\title{
Delta T: Polynomial Approximation of Time Period 1620-2013
}

\author{
M. Khalid, Mariam Sultana, and Faheem Zaidi \\ Department of Mathematical Sciences, Federal Urdu University of Arts, Sciences \& Technology, Karachi 75300, Pakistan
}

Correspondence should be addressed to M. Khalid; khalidsiddiqui@fuuast.edu.pk

Received 26 May 2014; Accepted 19 June 2014; Published 9 July 2014

Academic Editor: Josep M. Trigo-Rodríguez

Copyright (C) 2014 M. Khalid et al. This is an open access article distributed under the Creative Commons Attribution License, which permits unrestricted use, distribution, and reproduction in any medium, provided the original work is properly cited.

\begin{abstract}
The difference between the Uniform Dynamical Time and Universal Time is referred to as $\Delta T$ (delta $T$ ). Delta $T$ is used in numerous astronomical calculations, that is, eclipses, and length of day. It is additionally required to reduce quantified positions of minor planets to a uniform timescale for the purpose of orbital determination. Since Universal Time is established on the basis of the variable rotation of planet Earth, the quantity $\Delta T$ mirrors the unevenness of that rotation, and so it changes slowly, but rather irregularly, as time passes. We have worked on empirical formulae for estimating $\Delta T$ and have discovered a set of polynomials of the 4 th order with nine intervals which is accurate within the range of \pm 0.6 seconds for the duration of years 1620-2013.
\end{abstract}

\section{Introduction}

The expression "timescale" is quite frequently used in astronomical contexts. To define it in astronomical terms, it may be put as a way of measuring time based on a particular periodic natural phenomenon. Two main distinct groups of timescales are used in astronomy. The first group of timescales is based on second which are known as International Atomic Time (IAT). It is the standard for the SI (System International) second. The SI-based timescales are comparatively new in the history of timekeeping, since they depend on atomic clocks that were first put to regular use in the 1950s era. Prior to that, all timescales were associated somewhat with the rotation of the Earth.

Timescales that rely on the rotation of the Earth are used for astronomical purposes as well. A relevant example would be a telescope pointing that relies on the geographic orientation of the observer. Universal Time UT mostly refers to the specific timescale UT1. Historically, Universal Time (earlier known as the Greenwich Mean Time) has been achieved from Greenwich sidereal time using a general expression. However, UT is not fit for the computation of positions of the Moon, Sun, and planets using gravitational theories of their respective movements. Such theories prohibit variations in the rate of the rotation of Earth on its axis. Modern astrodynamical theories of the motions of the Sun, the Moon, and the planets are based on an evenly increasing and uniform timescale referred to as Terrestrial Time TT. TT runs a little ahead of UT1 (a refined measure of mean solar time at Greenwich) by an amount known as delta $T=T T-U T 1$. As Earth's rotation does not decelerate at a uniform rate, nontidal effects make it inconceivable to predict the precise values of $\Delta T$ in the distant past or remote future. Unfortunately, estimating the standard error in $\Delta T$ before $1600 \mathrm{AD}$ is a tough task. It depends on several factors including the accuracy of determining $\Delta T$ from previous eclipse records and designing the models of physical processes creating changes in Earth's rotation.

In the recent past, various polynomial representations for the $\Delta T$ values for the last few centuries have been suggested by Meeus [1], Islam et al. [2], and Meeus and Simons [3]. In this paper, we aim to present set of the 4 th degree polynomials for delta $T$ with the least possible absolute error within \pm 0.6 seconds for the duration of 1620-2013.

\section{Literature Review}

Proving that the Earth rotates was no easy task for the scientists of former times. This problem goes back to the 17th century, when Halley [4] found that quadratic terms had to be added to the Moon's mean longitude to match the times recorded for ancient eclipses. Laplace [5] announced that the acceleration term was due to perturbations from the Earth's orbital eccentricity. Adams [6] then determined 
that Laplace had not included many higher order terms, which reduced Laplace's final result to about half of Halley's empirical value. To explain the source for the remaining observed effect, Ferrell [7] and Delaunay [8] independently assigned this discrepancy to tidal interactions between the Earth and the Moon. Newcomb [9] analyzed variations of the Earth's rotation to explain some of the lunar residuals, but he could not obtain verification of these variations from inner planet data (Newcomb [10]).

In the latter part of the 19th century, Chandler discovered minute variations in Earth's axis of rotation or polar wobble (Chandler [11, 12]). Newcomb then announced that these, and possibly other irregular variations of the Earth's rotation, might be an explanation of the residuals in the lunar mean longitude (Newcomb [10]).

In all these cases, calculated positions were ahead of the observed positions by amounts proportional to the frequencies. Astronomers came to believe that discrepancies in ephemerides were not due to errors in the expressions for the mean longitude but were due to unmodeled irregularities and a deceleration of the Earth's rotation, on which UT depends. Spencer Jones [13] examined residuals in the mean longitudes of the Sun, Moon, and two other planets and then concluded that the error was due to a slow deceleration in Earth's rotation (Spencer Jones [14]). Since Newton's theory of gravitation requires a nonaccelerating uniform timescale for the computation of orbital motions and because the Earth's rotation was assumed to be decelerating, astronomers thought a timescale determined by the orbits themselves would be the uniform scale they needed. Thus, their proposition was a timescale called Ephemeris Time (ET), based on orbital motions, to be used for all dynamical calculations. In 1984, ET was replaced by Terrestrial Dynamical Time (TDT) as an independent argument for apparent geocentric ephemerides. The unit of TDT is a day of $86400 \mathrm{SI}$ seconds at mean sea level. For practical purposes, TDT is TAI + 32.184 seconds. In 1991, TDT was renamed Terrestrial Time (TT). TT is considered to be a uniform timescale and used as the time argument for the predictions of the astronomical events in dynamical theories (Seidelmann [15]; Islam et al. [2]).

Eclipse predictions are computed in TDT. To convert $T D T$ predictions to $U T$, the difference between TDT and UT1 must be in our knowledge. This parameter is known as $\Delta T=T D T-U T 1$ or delta $T$.

The mathematical modeling of the Earth's delta $T$ has been ongoing for some time and there are multiple models to choose from. Before the advancement of AD 1600, values of $\Delta T$ had to rely on historical records of naked-eye observations of eclipses and occultations. Stephenson and a few other researchers have identified hundreds of these types of observations in early European, Middle Eastern, and Chinese annals, manuscripts, canons, and records. Moreover, some of these scientists have fit a lot of records with simple polynomials to achieve best fits for describing the value of $\Delta T$ for the centuries occurring before AD 1600 (Morrison and Ward [16]; Stephenson and Fatoohi [17]; Stephenson and Fatoohi [18]; Stephenson and Morrison [19]; Stephenson and Morrison [20]; Stephenson [21]; Morrison and Stephenson [22]; Steele and Stephenson [23]). Despite their relatively low precision and inaccuracy, these data represent our only record of the value of $\Delta T$ during the past several millennia.

Close-to-accurate values of $\Delta T$ only exist sometime after the invention of the telescope (1610). A careful analysis of telescopic timings of stellar occultation by the Moon permits the direct measurement of $\Delta T$ during this time period. In fact, all values of $\Delta T$ before 1955 depend on observations of the Moon, either via solar eclipses or via lunar occultations (Seidelmann [15]). Nowadays, UT is representative of the observed rotation angle of the Earth relative to an inertial reference frame formed by extragalactic radio sources. Its measurement, with the help of several observatories, is coordinated by the International Earth Rotation and Reference Systems Service (IERS).

Numerous polynomial representations for the $\Delta T$ values of the last few centuries have been suggested in the recent past to evade the need of incorporating lengthy tables in a computer programs. Meeus [1] presents a 12th order polynomial liable for the time span of 1800-1997 with the maximum absolute error of 2.3 seconds and two 9th or 10th order polynomials covering a similar time span with a maximum absolute error of 1.069 SI seconds (Meeus [1]). Montenbruck and Pfleger [24] provide seven 3rd order polynomials to accommodate the period between 1825 and 2000 with maximum absolute error of 2.13 SI seconds. Meeus and Simons [3] give fourth-order polynomials with eight segments to cover the period between 1620 and 2000 with maximum absolute error of 3.2 SI seconds (Meeus and Simons [3]; Islam et al. [2]). Islam et al. [2] presented eight 4 th degree polynomials to the entire curve of $\Delta T$ with precisely the same intervals as were used by Meeus and Simons [3] compromising maximum absolute error of \pm 0.7 seconds on time span from 1620 to 2000 AD.

\section{New Proposed Polynomial Approximation}

The data used in this research was excerpted from the pages $K 8$ and $K 9$ of the Astronomical Almanac published by the Nautical Almanac Offices of the US Naval Observatory and the Bulletins issued by the International Earth Rotation Service, IERS, Paris. For a new set of polynomials, we apply method of the least square on the curve of delta $T$ to get appropriate polynomials for epoch 1620-2013 (Figure 1). The quantity $u$, as defined by Meeus and Simons [3], is

$$
u=k+\frac{(\text { year }-2000)}{100} .
$$

In this equation, the purpose of the $k$ quantities is basically to make the independent variable $u$ as small as possible during a certain interval of time, so we simply call it the "scaling factor."

The empirical formulae that we found here can calculate the value of $\Delta T$ at any instant of the years 1620-2013 $\mathrm{AD}$. The 4 th degree polynomial approximation of $\Delta T$ is

$$
\Delta T=a_{\mathrm{o}}+a_{1} u+a_{2} u^{2}+a_{3} u^{3}+a_{4} u^{4},
$$

where $a_{0}, a_{1}, a_{2}, a_{3}$, and $a_{4}$ are coefficients of respective polynomials taken in seconds, given in Table 1. 
TABLE 1: Values of scaling factor $k$ with coefficients of the 4 th degree polynomial.

\begin{tabular}{lccccccc}
\hline Duration & $k$ & $a 0$ & $a 1$ & $a 2$ & $a 3$ & $a 4$ & Max. err. \\
\hline $1620-1672$ & 3.670 & 76.541 & -253.532 & 695.901 & -1256.982 & 627.152 & 0.5709 \\
$1673-1729$ & 3.120 & 10.872 & -40.744 & 236.890 & -351.537 & 36.612 \\
$1730-1797$ & 2.495 & 13.480 & 13.075 & 8.635 & -3.307 & -128.294 \\
$1798-1843$ & 1.925 & 12.584 & 1.929 & 60.896 & -1432.216 & 3129.071 & 0.5989 \\
$1844-1877$ & 1.525 & 6.364 & 11.004 & 407.776 & -4168.394 & 7561.686 \\
$1878-1904$ & 1.220 & -5.058 & -1.701 & -46.403 & -866.171 & 5917.585 & 0.4643 \\
$1905-1945$ & 0.880 & 13.392 & 128.592 & -279.165 & -1282.050 & 4039.490 \\
$1946-1989$ & 0.455 & 30.782 & 34.348 & 46.452 & 1295.550 & -3210.913 \\
$1990-2013$ & 0.115 & 55.281 & 91.248 & 87.202 & -3092.565 & 8255.422 & 0.5410 \\
\hline
\end{tabular}

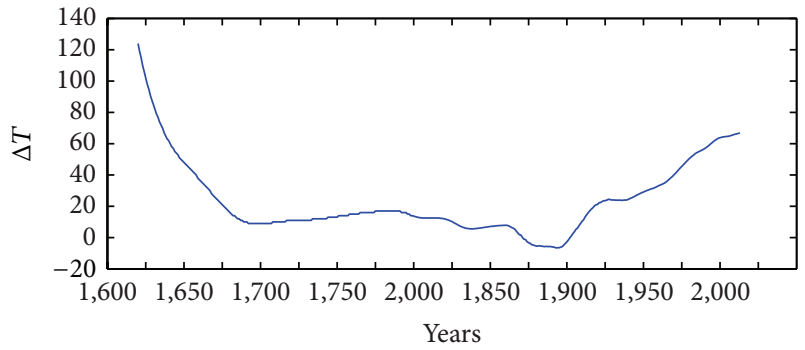

FIgURE 1: Curve shows variation in delta $T$ with epoch 1620-2013.

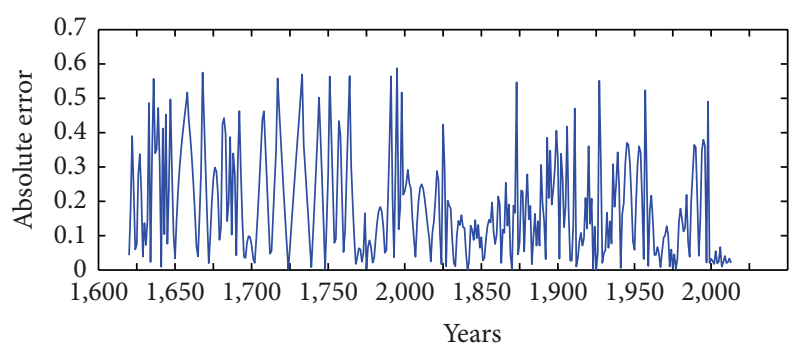

Figure 2: Graph shows absolute variation in $\Delta T$ (the 4 th degree polynomial) with epoch 1620-2013.

\section{Discussion of Results and Conclusion}

The value of scaling parameter $k$ and coefficients of the 4th degree polynomial with nine intervals that we have calculated are displayed in Table 1. It is clear from the table that the values of $k$ and coefficients are only valid for adjacent intervals. We choose the 4 th degrees polynomials in order to compare our results with previous approximations (Meeus and Simons [3] and Islam et al. [2]).

Figure 2 shows error analysis of the 4th degree polynomial with respect to its nine segments. Error analysis revealed that, in the 4 th degree polynomial, minimum error of 0.000168 occurs in the year 1712, while maximum error of 0.598961 appears in the year 1692 .

The maximum error found in this study is so minute that it turns out to be less than all sets of polynomials presented in Montenbruck and Pfleger [24], Meeus and Simons [3], and Islam et al. [2]. Therefore, it is concluded that a set of nine fourth degree polynomials has proven to be the most appropriate set covering a time span of 1620-2013 AD with \pm 0.6 seconds of accuracy. The time accuracy of astronomical events can predict the timescale in a much better fashion with this polynomial approximation. The uses include the solving of predicaments ranging from astronomical software to eliminating the steps in the search of the long table of the observed values of $\Delta T$ during the aforementioned period.

\section{Conflict of Interests}

The authors declare that there is no conflict of interests regarding the publication of this paper.

\section{Acknowledgments}

The authors would like to thank Ms. Wishaal Khalid and other, anonymous, reviewers for their valuable comments and suggestions for the improvement of the quality of the paper.

\section{References}

[1] J. Meeus, "The effect of delta T on astronomical calculation," British Astronomical Association, vol. 108, no. 3, pp. 154-156, 1998.

[2] S. Islam, M. Sadiq, and Q. Shahid, "Assessing polynomial approximation for delta T," Journal of Basic \& Applied Science, vol. 4 , no. 1, pp. 1-4, 2008.

[3] J. Meeus and L. Simons, "Polynomial approximation of Delta T," British Astronomical Association, vol. 110, pp. 323-324, 2000.

[4] E. Halley, "Some account of the ancient state of the city of Palmyra, with short remarks upon the inscriptions found there," Philosophical Transactions, vol. 19, no. 215-235, pp. 160-175, 1695.

[5] P. S. Laplace, Mécanique céleste, Memoires de l'Academie des Sciences de Paris, 1786.

[6] J. C. Adams, "On the secular variation of the moon's mean motion," Philosophical Transactions of the Royal Society A, vol. 143, pp. 397-406, 1853.

[7] W. Ferrell, "Note on the influence of the tides in causing an apparent secular acceleration of the moon's mean motion," Proceedings of the American Academy of Arts and Sciences, vol. 6, pp. 379-392, 1864.

[8] C. E. Delaunay, "Memoire sur une nouvelle méthode pour la determination du movement de la lune," Comptes Rendus de l'Académie des Sciences, vol. 61, 1865. 
[9] S. Newcomb, Researches on the Motion of the Moon, Part I, Washington Observations for 1875 (Washington: US Gov. Printing Off.), 1878.

[10] S. Newcomb, "Researches on the motion of Moon: part II," Astronomical Papers of the American Ephemeris and Nautical Almanac, vol. 11, 1912.

[11] S. C. Chandler, "On the variation of latitude ,I," The Astronomical Journal, vol. 11, no. 248, pp. 59-61, 1891.

[12] S. C. Chandler, "On the variation of latitude VI," Astronomical Journal, vol. 12, pp. 65-72, 1892.

[13] H. Spencer Jones, "The rotation of the earth, and the secular accelerations of the sun, moon and planets," Monthly Notices of the Royal Astronomical Society, vol. 99, pp. 541-558, 1939.

[14] H. Spencer Jones, “The rotation of the earth," Monthly Notices of the Royal Astronomical Society, vol. 87, pp. 4-31, 1926.

[15] P. K. Seidelmann, Explanatory Supplement to the Astronomical Almanac, pp. 39-93, University Science Books, Mill Valley, Calif, USA, 1992.

[16] L. V. Morrison and C. G. Ward, "Analysis of transit of Mercury," Monthly Notices of the Royal Astronomical Society, vol. 173, pp. 183-206, 1975.

[17] F. R. Stephenson and L. J. Fatoohi, "Solar and lunar eclipse measurements by medieval muslim astronomers I. Background," Journal for the History of Astronomy, vol. 25, p. 99, 1994.

[18] F. R. Stephenson and L. J. Fatoohi, "Solar and lunar eclipse measurements by medieval muslim astronomers I I. Observations," Journal for the History of Astronomy, vol. 26, pp. 227-236, 1995.

[19] F. R. Stephenson and L. V. Morrison, "Long-term changes in the rotation of the Earth: 700 B.C. to A.D. 1980," Philosophical Transactions of the Royal Society of London A, vol. 313, pp. 47-70, 1984.

[20] F. R. Stephenson and L. V. Morrison, "Long-term fluctuations in the Earth's rotation: 700 BC to AD 1990," Philosophical Transactions of the Royal Society of London A, vol. 351, pp. 165202, 1990.

[21] F. R. Stephenson, Historical Eclipses and Earth's Rotation, Cambridge University Press, Cambridge, UK, 1997.

[22] L. V. Morrison and F. R. Stephenson, "Historical values of the Earth's clock error Delta T and the calculation of eclipses," Journal for the History of Astronomy, vol. 35, no. 2, pp. 327-336, 2004.

[23] J. M. Steele and F. R. Stephenson, "Astronomical evidence for the accuracy of clocks in Pre-Jesuit China," Journal for the History of Astronomy, vol. 29, pp. 35-48, 1998.

[24] P. Montenbruck and T. Pfleger, Astronomy on the Personal Computer, Springer, Berlin, Germany, 2000. 

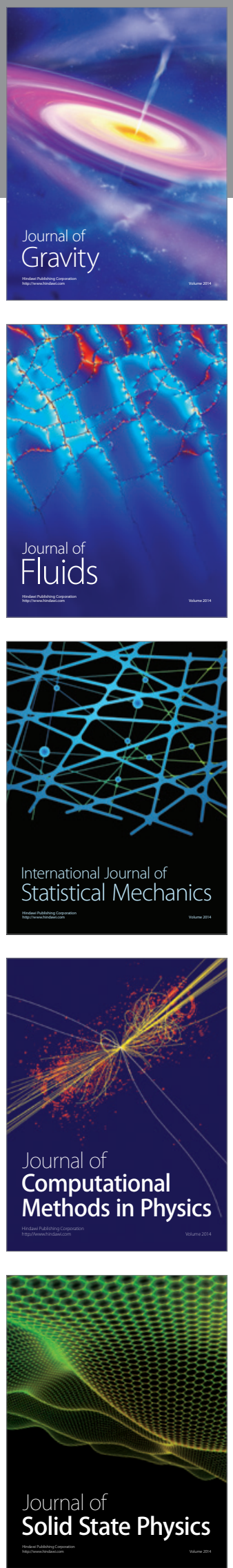

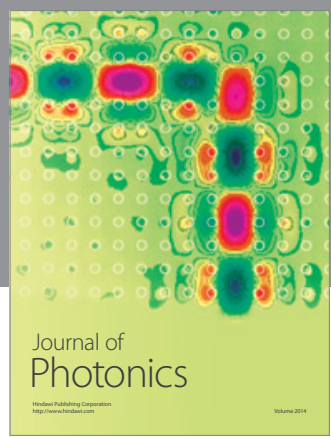

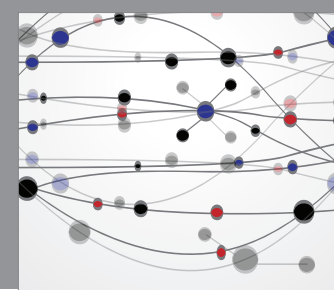

The Scientific World Journal

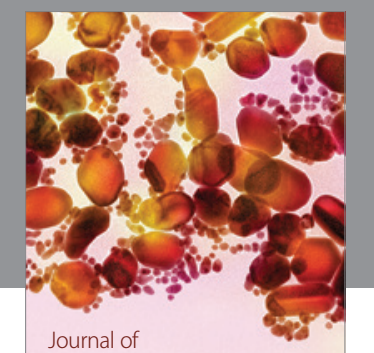

Soft Matter
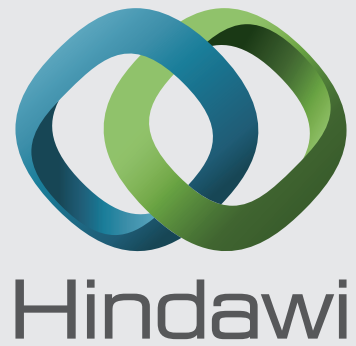

Submit your manuscripts at

http://www.hindawi.com
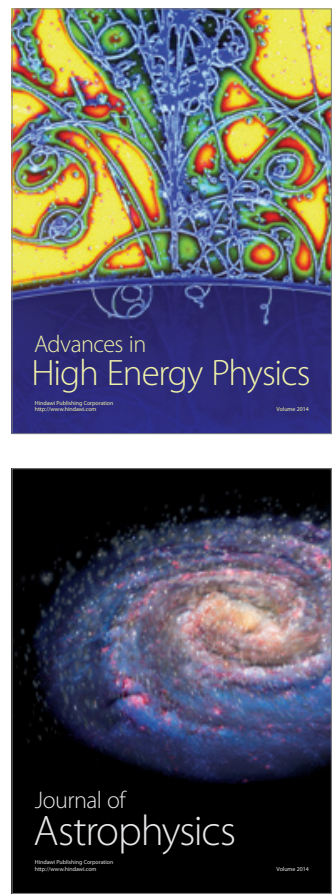
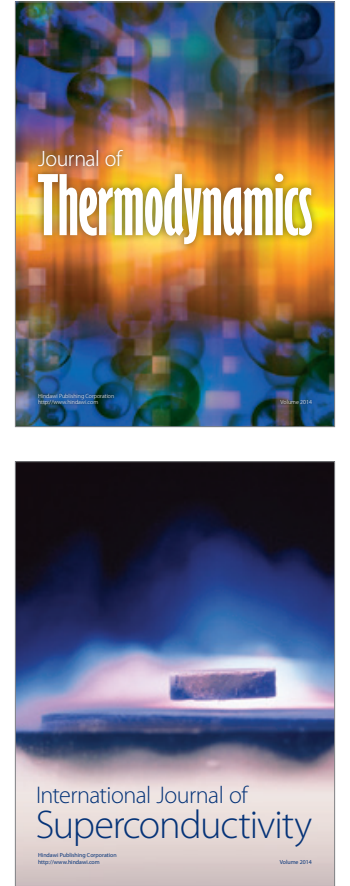
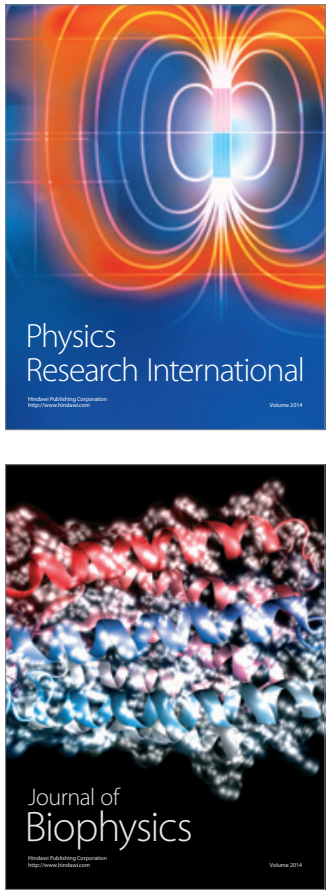
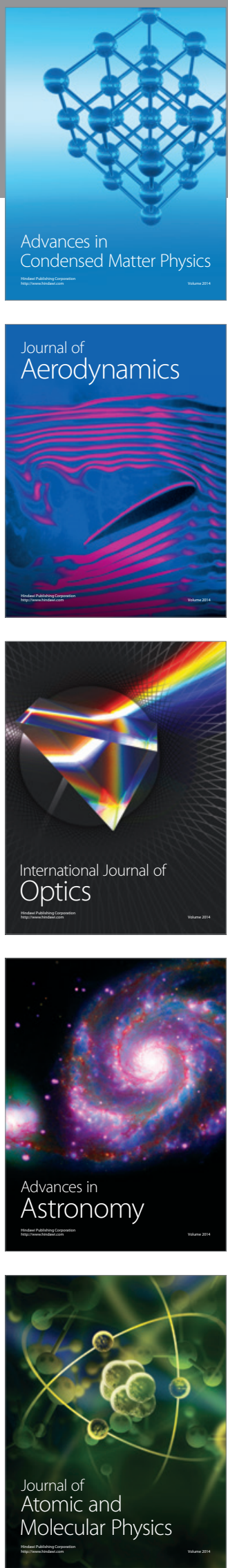